\title{
Combining Web Technology and Mobile Phones to Enhance English Literacy in Underserved Communities
}

\author{
Hatem Alismail \\ Carnegie Mellon University \\ 5000 Forbes Avenue \\ Pittsburgh, PA 15213 USA \\ $+1-412-268-1289$ \\ halismai@cs.cmu.edu \\ Anthony Velázquez \\ Carnegie Mellon University \\ 5000 Forbes Avenue \\ Pittsburgh, PA 15213 USA \\ +1-412-268-1289
}

anthony.velazquez@gmail.com

Ermine A. Teves

Carnegie Mellon University 5000 Forbes Avenue

Pittsburgh, PA 15213 USA

+1-412-268-1289

eteves@cs.cmu.edu

Bradley Hall

Carnegie Mellon University 5000 Forbes Avenue

Pittsburgh, PA 15213 USA

$+1-412-268-1289$

hallbt@gmail.com
Aysha Siddique

Carnegie Mellon University in Qatar

P.O. Box 24866

Doha, Qatar

+974-4454-8641

aysha.siddique@gmail.com

M. Beatrice Dias

Carnegie Mellon University 5000 Forbes Avenue

Pittsburgh, PA 15213 USA

+1-412-268-1289

beadias@cmu.edu

Rotimi Abimbola

Carnegie Mellon University 5000 Forbes Avenue

Pittsburgh, PA 15213 USA

+1-412-268-1289

rotimi.abimbola@gmail.com

M. Bernardine Dias

Carnegie Mellon University 5000 Forbes Avenue

Pittsburgh, PA 15213 USA

+1-412-268-9365

mbdias@ri.cmu.edu

\author{
M. Freddie Dias \\ Carnegie Mellon University \\ 5000 Forbes Avenue \\ Pittsburgh, PA 15213 USA \\ +1-412-268-1289 \\ mfdias@ri.cmu.edu \\ Sarah Belousov \\ Carnegie Mellon University \\ 5000 Forbes Avenue \\ Pittsburgh, PA 15213 USA \\ +1-412-268-7147 \\ sarahtbw@ri.cmu.edu \\ Daniel Nuffer \\ Carnegie Mellon University \\ 5000 Forbes Avenue \\ Pittsburgh, PA 15213 USA \\ +1-412-268-1289 \\ nuffer@gmail.com
}

\begin{abstract}
Despite the importance of literacy to most aspects of life, underserved communities continue to suffer from low literacy rates; especially for globally prevalent languages such as English. This is particularly problematic since English literacy is an important skill for increased access to income generation and higher education opportunities in these communities. Teaching English in underserved communities can be challenging due to a variety of obstacles. In this paper we report on our work with a primary school in Tanzania, with migrant workers in Doha, and with a school for the deaf in the USA to address some of these challenges using web technology and mobile phones. We developed a web-accessible content authoring tool that teachers
\end{abstract}

Permission to make digital or hard copies of all or part of this work for personal or classroom use is granted without fee provided that copies are not made or distributed for profit or commercial advantage and that copies bear this notice and the full citation on the first page. To copy otherwise, to republish, to post on servers or to redistribute to lists, requires prior specific permission and/or a fee.

ACM DEV'10, December 17-18, 2010, London, United Kingdom.

Copyright 2010 ACM 978-1-4503-0473-3-10/12... \$10 can use to create and modify English grammar exercises, and a simple game that students use on a mobile phone to practice the content uploaded from the authoring tool. Our solution is primarily intended to increase the motivation for practicing English grammar among students, and thereby enhance English literacy in underserved communities. Feedback from teachers and students in all of our field studies indicate this solution is affordable and has significant potential to enhance the English classroom and homework experience.

\section{Categories and Subject Descriptors}

K.3.1 [Computers and Education]: Computer Uses in Education - collaborative learning, computer assisted learning, distance learning.

\section{General Terms}

Design, Experimentation, Human Factors.

\section{Keywords}

Design, Experimentation, Human Factors. 


\section{INTRODUCTION}

Globalization has propelled the need for a common language that can be used for communicating across international boundaries. English has become one of the most important languages used in international business, technology, and everyday life. Therefore, some level of fluency in English becomes a pre-requisite for many employment opportunities around the world. In today's globalized world, the ability to communicate in English can outweigh experience and other skills in priority when considered for several career advancement and employment opportunities. Additionally, fluency in English can allow a person to acquire a variety of skills where the medium of instruction is often English (for example, computer courses), and thus further enhance opportunities for career advancement. Conversely, possessing limited or no English language skills, due to a lack of opportunities and resources in education, inadequate access to qualified teachers, and/or difficulties in grasping the nuances of the language, can disadvantage individuals in many populations. This work seeks to improve this situation for underserved communities in an affordable, engaging, and practical manner through the combined use of web technology and mobile phones.

Today, mobile phones contain the most accessible and ubiquitous computers. Almost everyone, in both developed and developing communities, has frequent access to a mobile phone. People use their mobile phones for many tasks beyond communication, including storing and accessing videos, music, and pictures, and playing games. Mobile phone games are popular among adults and children alike. Some of the clear merits of mobile phones as tools in underserved communities compared to other technologies include affordability, ubiquity, portability, increasing computational power, and low power consumption fueled by battery power (does not require continuous access to a reliable power grid). Our work therefore seeks to exploit the mobile phone as a tool to improve English literacy in underserved populations. To this end we intend to design a simple game that can run on low-end mobile phones, combined with a collaborative web-based platform that allows the authoring of educational content that can be uploaded to the game. To our knowledge, this combination of an educational mobile-phone game with a web-based content authoring tool is a novel contribution to the field.

In this paper, we explain the details of our English literacy tool, describe the preliminary testing of this tool in three different underserved communities, and demonstrate the potential of this tool in terms of both scalability and impact in a variety of settings. We conclude with a discussion of the limitations of the current tool and how these limitations will be addressed in future work.

\section{RELATED WORK}

The literature on computer assisted learning is extensive and diverse. A range of technology devices and platforms has been explored in the context of literacy tools development, with laptop and desktop computers being the most widely used platforms. A great example of computer use for education is Project LISTEN [10]. LISTEN aims to improve children's reading skills through an automated tutor. Children can choose stories they want to read and the tutor "listens" and intervenes when the child makes a mistake, gets stuck, or faces difficulty reading certain sections or words. LISTEN was also deployed in Ghana and showed promising results [8]. In a similar project [2], the authors describe another computer assisted learning project targeting English literacy and mathematics. The authors report on experiments conducted in urban India and show improvements on test scores after a yearlong study. While traditional computer based tools can be very powerful, their main drawback is the associated cost and the need for a stable source of electricity. Many students in underserved communities do not have access to computers that are capable of running these applications. Even in situations where such computers might be available, challenges such as long and frequent power failures prevent their regular use. Although several groups are working to serve educational needs in developing communities through lower-cost and more robust computers [11], and through different methods for sharing a single computer among several students [12], these solutions are not yet widespread.

In contrast, mobile phones are relatively cheap computing devices that are commonly found in most communities. Recently, several groups have begun to explore the use of mobile phones for a variety of applications including education in underserved communities. One example is a pilot study that used cell phones to improve children literacy as well as to engage their parents in the process [13]. However, working with the parents at the level of this study is unlikely to scale. Horowitz et al. [4], show a successful mobile phone based effort tested with children from low and high income families. However, the study was only conducted in the United States. Another example of mobile phone use in literacy tools development is MILLEE, or Mobile and Immersive Learning for Literacy in Emerging Economies [1]. MILLEE is one of the few efforts that utilize the potential of cell phones for developing literacy tools in underserved communities. With an initial focus on India, MILLEE develops mobile games and applications for school-aged children to improve various literacy skills. They create their own games adapted from popular culturally relevant games to introduce new English vocabulary and grammar to children through a receptive-practice-activation cycle wherein students are taught new concepts, allowed to practice these concepts, and then tested on the material they were presented.

MILLEE's goals are similar to our work; however, there are significant differences. First, games used in MILLEE's project are informed by experts on teaching English skills, focused on the students, and act as stand-alone tools that are used outside the school environment. In contrast, our work is designed to complement classroom activities, and seeks to benefit teachers as well as students. Teachers are able to alter the content of the game and can customize it to fit their teaching needs. Moreover, MILLEE is set up as a social innovation project that is modeled for cost recovery with proprietary software. Our work will be released under an Open Source license that can be easily shared by members of the community with no cost to them.

Yet, another great platform for literacy tools development is the Internet. The World Wide Web contains a large number of applications and games targeted at improving literacy in many aspects. However, for underserved communities, a full utilization of the Internet has the same limitations and challenges associated with using computers. In fact, stable access to the Internet is a large cost burden in many cases. In this work, we propose the use of web applications as a content authoring tool to be used in 
conjunction with the mobile phone game. The application does not require constant Internet access and can be run on a browser offline. When Internet access is available, the application can be used as a medium of collaboration between experts in teaching and teachers in underserved communities to help each other create better content. To our knowledge, the use of web applications in this context to improve English literacy in underserved communities is novel.

Before delving into the details of our research methodology and proposed approach, we next introduce the three communities we partnered with to test our work.

\section{COMMUNITIES}

We identified three groups who were interested in using our English literacy tool. They are primary school students in a lowresource community, low-skilled migrant workers, and middle school deaf students; three populations that face different challenges in learning English. We describe each of these communities in greater detail next.

\subsection{Primary School in Tanzania}

Our first user group was primary school students at the Mlimani Primary School in Dar es Salaam, Tanzania. The work was part of the iSTEP internship in 2009 [1]. Mlimani includes approximately 1,500 students, many of whom are from families with limited resources. There were approximately 42 active teachers at the school and most of them taught a variety of subjects. Each class comprised of 60 students, on average. At the time of our work with them, our partner school did not have electricity or any computing technology, and in general had access to very few educational materials and limited infrastructure.

Although Kiswahili is the national language in Tanzania, English literacy is an important prerequisite for both educational and employment advancement opportunities. For secondary schools and other institutions of higher learning, English is the medium of instruction. Kiswahili is used as the medium of instruction in all public primary schools, except in the case of English, which is taught as one subject out of nine in total. English education in primary schools is riddled with challenges, particularly since most teachers are not native English speakers.

\subsection{Migrant Workers in Qatar}

Our second user group was adult migrant workers with blue collar jobs in Qatar. This population is typically characterized by limited educational background, low wages, and jobs in the construction, domestic help, and service sectors. Their limited English skills create barriers in their career advancement since they perform poorly when communicating with supervisors and other authorities and cannot successfully negotiate their pay, vacation, and accommodation. Such problems lower their standard of living and make it difficult for them to assimilate and adjust into the foreign culture.

In 2010, we partnered with two institutions, the first being Reach Out To Asia (ROTA), a non-governmental charity organization in Qatar. ROTA started an Adult English Literacy program (RAEL) to teach basic and intermediate English skills to immigrant laborers working with construction companies. The laborers volunteer to join the classes and are awarded certificates at the end of the eight week program. The second institution we partnered with was Carnegie Mellon University in Qatar (CMUQ) which employed immigrant workers as service attendants. Learning English skills would be highly beneficial to the service attendants since their job requires them to communicate with professors and students from different cultures where the common language is English.

\subsection{School for the Deaf in the USA}

Our third user group is comprised of middle school Deaf and hard-of-hearing students. We partnered with the Western Pennsylvania School for the Deaf (WPSD), a non-profit school which provides educational services and a complete extracurricular program to Deaf and hard-of-hearing individuals from birth through Grade 12. WPSD serves over 250 students from more than 100 school districts and 30 counties in Pennsylvania and is the largest comprehensive center for deaf education in the state.

Even though English is the primary language (other than sign language) for the majority of the students, they have trouble grasping it due to the stark structural differences between English and American Sign Language (ASL). The average deaf adult in the USA reaches only a 4th grade English reading level, and approximately $10 \%$ of deaf 18 year olds can read at or above an 8th grade level [7]. Deaf individuals usually have "severely limited vocabulary and lack knowledge of the complex syntax of English that is critical for combining sentences together into cohesive text" [5]. Their difficulties with communicating in English also add an extra layer of difficulty for deaf employees at work places that are shared by both deaf and hearing individuals, thus limiting their opportunities for career advancement.

\section{METHODOLOGY}

Developing an effective tool that can enhance English learning required us to understand the challenges faced by potential users of this tool when learning English, as well as their cultural and educational backgrounds, and their interests. This needs assessment process plays a critical role in developing technology that is practical, sustainable and relevant to a specific community.

\subsection{Needs Assessment}

In our needs assessment process, we first reach out to potential community partners and build relationships with appropriate members from each community (such as administrators, teachers, students and workers).

Building positive relationships with these members is a crucial step in gaining their trust and eliciting honest responses in the needs assessment process. We then interview as many members as possible to gather their relevant insights, opinions and suggestions according to their expertise. Since this work centers on improving English literacy skills, in addition to interviews, we also observed English classes to obtain a first-hand view of how classes are conducted and to interact with the students. We also met with experts on teaching English as a foreign language (EFL) and with their help we reviewed the relevant literature on best practices in EFL instruction.

Once the needs assessment process is complete, we compile and analyze the data. Based on the needs assessment data, we 
brainstorm possible solutions and present the solution ideas to appropriate community members. Once a solution is agreed upon, the technology development begins and field tests are conducted with appropriate members of the community. We collect feedback from the user groups and take this feedback into consideration for further iterations of the technology solution. Sustainability of the project is also discussed with relevant community members. Figure 1 demonstrates the needs assessment process. Next, we summarize the key findings from the needs assessment process from the three communities we worked with.

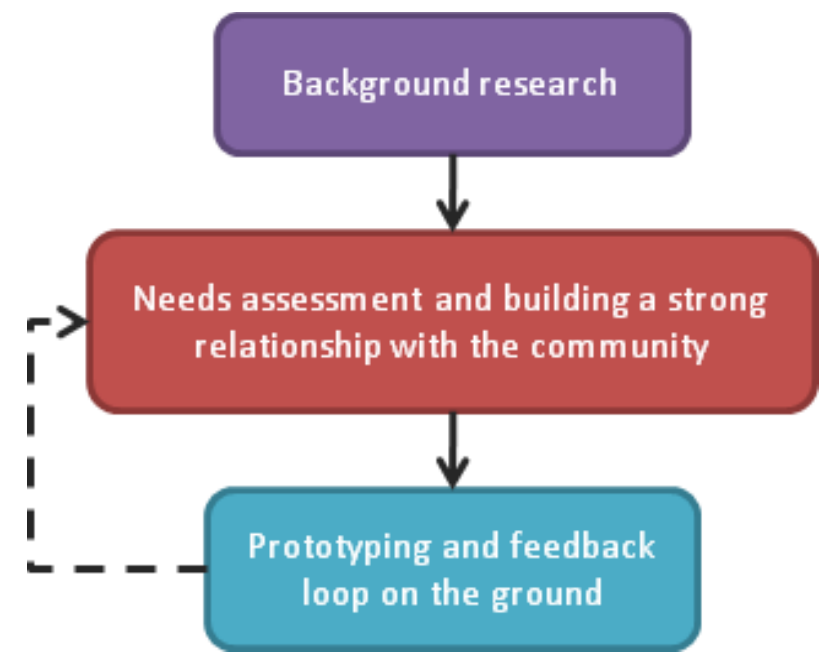

Figure 1. Summary of research methodology.

\subsection{Key Findings from Needs Assessment}

Our needs assessments process revealed several needs common to all of our user groups. A summary of the key findings are as follows:

- Many English grammar concepts are confusing to students, and they will benefit from more practice in articles, noncount verbs, and verb tenses.

- Students are confused by the meaning and use of homophones such as "too", "two" and "to".

- All of the users have access to mobile phones - some of them own phones individually and others have access to shared phones.

- Playing or watching sports is a favorite pastime of most participants in all of our user groups and football (soccer) was the most popular sport averaged across all users from the different communities.

- Users enjoyed graphical components of games.

- Users were motivated to play challenging games for longer durations.

- Users wanted to see their progress in a game so they could feel a sense of accomplishment when they complete different components of the game.

- Learning new vocabulary is a challenge for all of the user groups.
- Teachers wanted to benefit from the knowledge, expertise, and curricula of other teachers and experts in instruction of English as a Foreign Language (EFL), but wanted to have control over the content presented to their students.

Based on the findings of our needs assessment process, we iterated on solution concepts with our community partners and designed the English literacy tool described next.

\subsection{Solution Overview}

Our solution consists of two parts: (1) an online content authoring tool for use by teachers, and (2) a quiz-based educational game for the students to play on mobile phones. The content authoring tool was created to give teachers control of the game's content and allow them to customize it based on their curriculum and classroom. For example, teachers might upload different exercises for each week of classes that compels students to practice the particular concepts to be taught that week. The game was created to motivate students to practice grammar exercises uploaded from the content authoring tool. We cater to the varying levels of English literacy skills among students by dynamically scaling the difficulty level of the exercises based on student performance. The dynamic adjustment of difficulty level allows us to challenge students with stronger English literacy skills without discouraging students who are at a lower skill level. Next, we discuss our solution components in greater detail.

\section{THE CONTENT AUTHORING TOOL}

The content authoring tool (CAT) was designed for teachers to create, edit, and select content for students according to their lesson plans. Intuitive use was the main consideration during the design and development of the tool, because some teachers did not have a lot of experience working with computers. The tool guides the teachers through the steps needed to create questions through a user-friendly interface.

\subsection{Process for Entering Content}

The process for teachers to enter content using the CAT is as follows. First, the teacher needs to select a question type, and if applicable, a "category" used to automatically generate a sensible set of multiple-choice answers. Two aspects of the English curriculum that students often struggle with are grammar and vocabulary. Typically, there is a small set of common answers for a larger set of questions. For example, common answers include: have/has/had, she/he/they/it. Hence, the possible answers that students can select are drawn from a pool of limited possibilities. We refer to different groups of answers as categories. For example, the answer "school" belongs to the "places" category, while "and" belongs to the "conjunctions" category. We store a list of possible solutions from each category so the CAT can intelligently automate the selection of answer choices for multiple-choice questions. This approach makes authoring questions an easier task.

The CAT also provides teachers with the option to add, edit, and delete categories. To add a category, the teacher specifies the name of the category and the answer options within that category. There are also examples of questions where having a selection of answers from a pre-defined category does not make sense. Therefore, the teacher has the option of not specifying a category 
and manually entering the answer choices. However, if the same answer choices can be used for other questions as well, the teacher has the option to create a category for those answer choices that allows the creation of multiple questions without having to re-enter the answer choices.

According to EFL experts, the use of images enhances the students' ability to learn a language. Thus, the CAT can also support the use of images in questions in two formats: image question with text answer, and text question with image answers.

Based on advice from EFL experts and sample questions from teachers, the CAT was created to support the following five types of questions:

1. Writing questions

2. Multiple choice questions with categories

3. Multiple choice questions with user defined options

4. Image questions with text answers

5. Text questions with image multiple choice answers

An example of each type of question is shown in Figures 2, 3, 4, 5,6 , respectively.

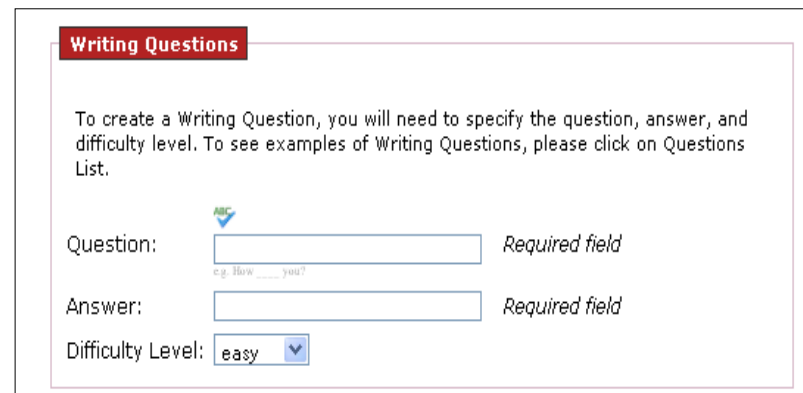

Example:

Question: What _- your name?

Answer: is

Figure 2. Writing question.

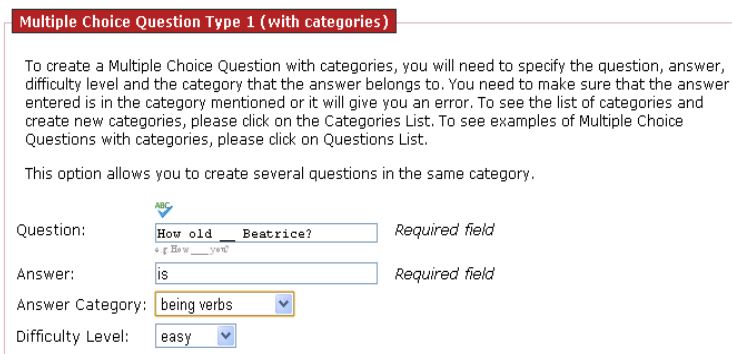

\section{Multiple Choice Question Type 1 (with categories)}

To create a Multiple Choice Question with categories, you will need to specify the question, answer, difficulty level and the category that the answer belongs to. You need to make sure that the answer create new categories, please click on the Categories List. To see examples of Multiple Choice Questions with categories, please click on Questions List.

This option allows you to create several questions in the same category.

Questionicher

Answer Category: being verbs $v$

Difficulty Level: easy $v$

Example:

Question: How old _- Beatrice?

Answer: is

Category: being verbs

Figure 3. Multiple choice question with categories.

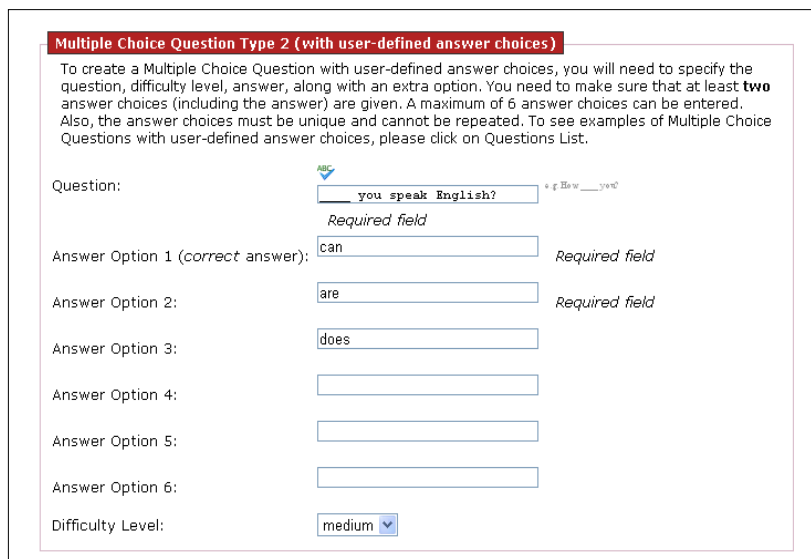

Example:

Question: you speak English?

Answer: can

Op 1: are

Op 2: can

Op 3: does

Figure 4. Multiple choice question with user defined options.

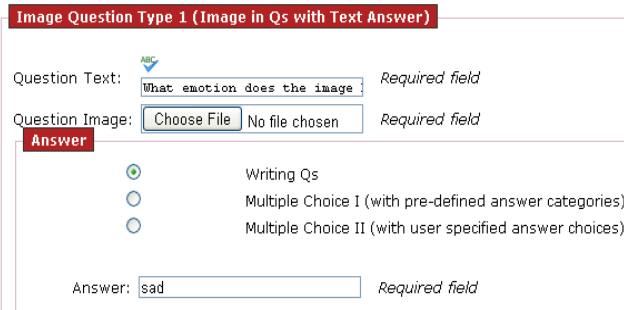

Example:

Question: What emotion does the picture below describe?

Question image:

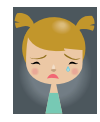

Answer: sad

Figure 5. Image question with text answer. 


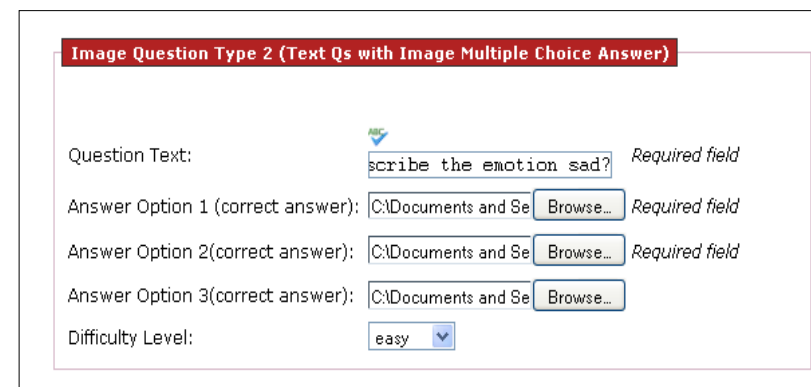

Example:

Question: Which image describes the emotion sad?

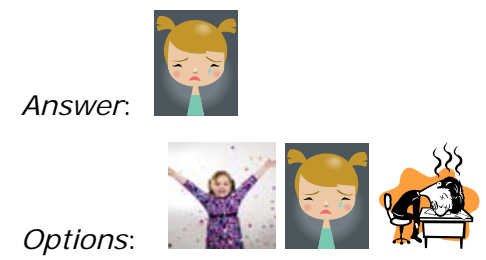

Figure 6. Text question with image answers.

Next, the teacher adds the relevant content by either typing new text, selecting existing options, or adding image files, and then selects the difficulty level of the question. The CAT spell checks the teacher's input and when the teacher selects "done” an XML content file is created and ready to be uploaded to a mobile phone. The XML question file can be uploaded to the phone using different methods and can be located anywhere in the phone memory. Depending on the phone, USB, infrared (IR), and Bluetooth are all viable options of uploading the file. Once the file is placed in the phone memory, it can be loaded into the game using the Options menu in the game's main screen.

\subsection{Database Backend to the CAT}

A MySQL backend to the CAT enables teachers to save questions entered into the database in appropriate tables, and also enables functionality such as editing and deleting questions. The XML file reproduces the list of all questions saved in the database. HTTP POST variables from the forms are processed by a PHP script and entered into the appropriate database based on the type of question.

\subsection{Content Transfer to Game from CAT}

The game's content (list of questions) is stored in a plain XML file that can be read by any text editor. For all text-only questions, the XML file generated by the CAT can be saved onto the phone and then can be added to the list of questions in the game by selecting the new file in the "Options" menu. For image questions, however, the images need to be copied separately and compiled into the game's binary file. This requires the help of a developer who would need to download the XML file and the corresponding images, copy them into the resources folder, and then compile a new binary file to be loaded onto the phone. The need to integrate images into the compiled game is due to security restrictions in JavaME.

\section{THE MOBILE PHONE GAME}

The primary goal of the mobile phone game is to provide additional motivation for students to practice their English literacy skills. In order to engage students, the game uses football penalty kicks in the form of graphical animations and appropriate sound effects. If the student answers a question correctly, he or she scores a goal and a cheering sound is played. Conversely, when the student answers incorrectly, a "goal-miss" animation and sound effects are displayed, and the student is encouraged to try again.

Portability to different platforms is a major concern when developing applications for mobile phones, especially in our case. The game was required to run on the various teachers' and students' mobile phones. Due to portability requirements, the game was implemented in Java ME [9] and the Light Weight User Interface Toolkit (LWUIT) [6] conforming to MIDP 2.0 and CLDC 1.1 specifications [3].

Most questions in the game are text-only, and based on selecting the right answer from a list of possible answers. For image questions with text answers, the form displays the question along with the question image and the list of answer options. This is done with the help of a LWUIT Container component inside the form. The image is displayed as a disabled button. For text questions with image answers, the form displays a question and then a list of images as answer options. This is also done with the help of LWUIT Button and Container components, with the Box Layout along the y-axis. The answer images are displayed as buttons, and to select the correct answer, the user has to click on the correct button.

The game consists of four main components: a scoring scheme, adaptive difficulty, an audio-visual interface, and the options menu. These components are described next.

\subsection{Scoring Scheme}

Since the game is a single-player game, we have implemented a "high-score" based scoring system. The score is computed based on the number of questions answered correctly weighted by their difficulty. Wrong answers do not lower the player's score, instead they add to the phone's score. The aim is that the student never "loses" and remains motivated to catch up to the phone's score.

\subsection{Adaptive Difficulty}

The game adjusts the difficulty level of the chosen questions based on the students' performance. Questions start at a medium level of difficulty and become easier or harder as the student progresses. In our implementation, a student has to consecutively answer five questions correctly in order to move to the next level of difficulty. If the student enters two wrong answers (even if they are not consecutive) the difficulty level of the game decreases by one unit. The difficulty levels of the questions are manually indicated by the teachers at the time of authoring.

\subsection{Visual and Audio Interface}

An audio-visual interface is essential to capture and retain the user's attention. However, care has been taken to reduce unnecessary distractions. If the student answers a question correctly, a football goal animation is displayed on the screen. 
The animation consists of a player attempting a penalty kick while the goalkeeper tries to defend the goal.

As shown in Figure 7, upon scoring the goal, an animated "goal" text image is shown with cheers and applause sounds. If the student answers the question incorrectly, a similar animation is displayed on the screen. However, in this case, the ball misses the goal or the goalkeeper catches the ball, and the animated text displayed reads "sorry" (for the primary school students in Tanzania, "sorry" was translated to Kiswahili) accompanied by relevant sound effects to encourage the student to try again and select the correct answer.

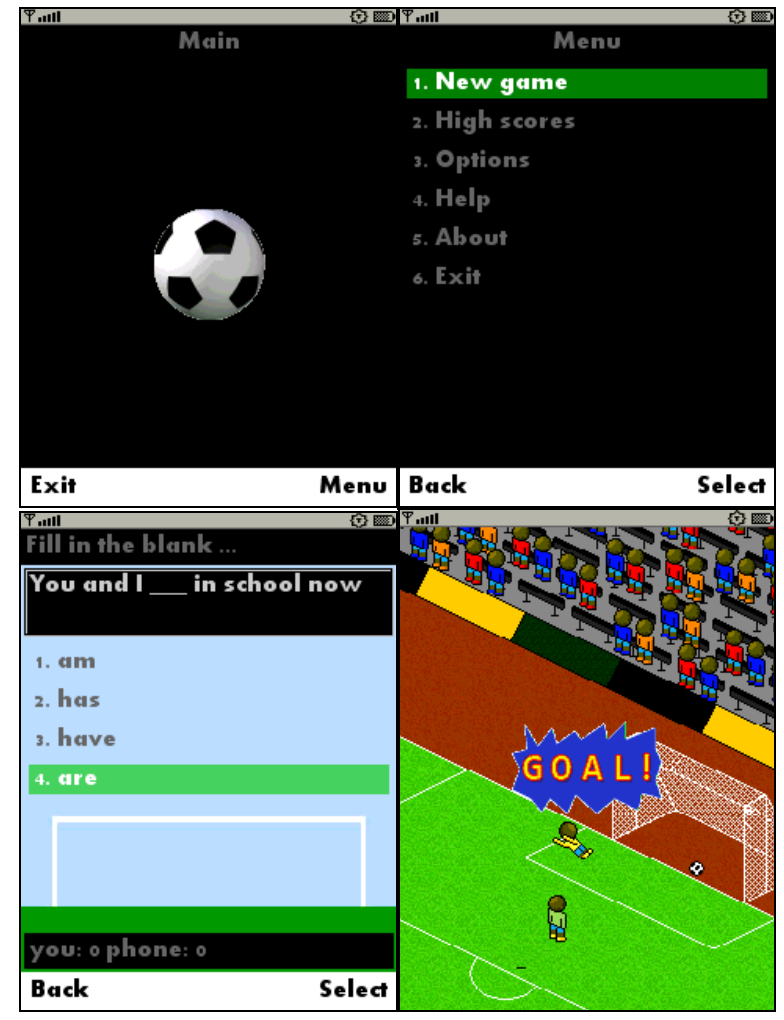

Figure 7. Screenshots of the mobile phone game.

\subsection{The Options Menu}

The options menu contains four main options:

1. Toggle on/off the audio components of the game

2. Switch between content files that come prepackaged with the game

3. Change the content file to a customized version located in the phone's memory

4. Reset the content file

The distinction between content files that come prepackaged with the phone vs. content created externally was necessary. For security reasons, Java ME does not allow modifying the internal data stored in the executable uploaded to the game and it does not allow the code to access the phone's memory without the user's permission. Hence, the game comes with a set of questions preloaded that does not require recompiling the executable. The third option starts a file browser to look for the customized content and acquires the user's permission necessary to access the file. Finally, the fourth option resets the main content file to a default file as a precaution in the event that an error occurs.

\section{USER FEEDBACK}

Once a complete version of the game was implemented, we tested the implementation in stages with teachers and students in the three user groups. The first versions of the CAT and mobile phone game were tested in the primary school in Tanzania. Feedback from the Tanzania pilot study was integrated into the work and significant improvements were made prior to subsequent tests with migrant workers in Qatar and the school for the deaf in the US. We start with discussing teachers' feedback, and then follow with the students' feedback.

\subsection{Teacher Feedback}

In all of the user groups where teachers were involved, we started by collecting feedback from the teachers.

\subsubsection{Primary School in Tanzania}

Teachers at the Mlimani School were first shown the game, and they agreed that it was intuitive and easy to use, which is critical when dealing with young learners. Also, teachers enjoyed the design of the game, especially the choice of football as the main theme. The lack of phones in the classroom to accommodate a large number of students was one of our concerns with this user group. However, teachers assured us that up to five students would be able to share the same phone. A useful suggestion by the teachers was to increase the font size used in the game to make it easier for students to read; especially in settings where multiple students are sharing a phone. Furthermore, teachers suggested that we should make sure the number of solutions provided as multiple choice options should match the students' exams and homework.

Next, we conducted a training session for teachers to instruct them on how to use the CAT. Training was conducted at a nearby university computer lab. We obtained permission for the teachers to use the computers in this lab free of charge. Teachers did not have any trouble creating new content using the CAT and uploading the content to the mobile phone game. Editing questions, however, was more challenging. The first version of the CAT had no feature to edit questions, and hence, teachers would have to edit the XML file in order to modify the questions. In addition, the questions were not saved in the CAT, and so teachers had to create new content for each game session. Based on the teacher's feedback we enhanced the editing capabilities of the CAT and added a database backend to the CAT to store the questions before testing with the other two user groups. Apart from editing, the teachers enjoyed using the CAT and creating content for the game. Finally, the automated tool for checking spelling in the CAT proved to be very helpful and corrected several mistakes the teachers made when creating content.

\subsubsection{Migrant Workers in Qatar}

In CMU-Q, the migrant workers were not in a classroom setting so we did not have the option of getting teacher feedback. The workers involved with ROTA were in a classroom setting where the instructors were undergraduate students who were well versed 
with using computers and hence had no problems using the CAT, playing the game, or uploading content from the CAT to the phones. These instructors updated the game content regularly and were able to provide initial feedback on the features of the CAT. They wanted to be able to select specific questions to use on the mobile phone game from the questions database so they could assign specific homework questions to the workers. At the time of this testing, all of the questions in the database got uploaded onto the mobile phone. The instructors also liked the ability to edit and delete questions to correct errors and avoid redundancy. Lastly, they preferred multiple choice questions with user defined options because it was more flexible and allowed them to meet the curriculum requirements more easily.

\subsubsection{School for the Deaf in the USA}

In our third user group, we demonstrated the game to a teacher and to three administrators at WPSD and they agreed that the game would be of interest and value to their students. Since students are not permitted to use their own phones in class, we agreed to loan eight phones to the teacher who was our primary contact at the school for testing the game during class. The focus of our pilot study was to evaluate the feasibility of the game in an in-class setting. Given time limitations, we did not evaluate the CAT with teachers in this user group but plan to do so in future field tests. In this pilot study, the teacher provided us with 10 groups of 10 questions each which we entered into the CAT and then uploaded to the phones. This saved the teacher some time and also ensured that all of the phones were functioning with the correct question files.

Although we did not evaluate the CAT with the teachers in this user group, their initial feedback was that the tool would be useful as a time-saver for the teacher. They often teach similar lessons year after year. Providing a way to enter questions in the CAT which they can use repeatedly with different classes allows them to save significant time. During the 10-day pilot study, the teacher who was our primary contact with the school observed many ways in which the game could be utilized in her classroom. For example, playing the game for 10 minutes at the start of each class focused the attention of the students on their lesson for the day. The game could also provide opportunities for competition and challenges between student peers. She also saw that it could be useful both inside and outside of the classroom, especially since some students live on campus but travel long distances to return home on the weekends and can play the phone game during such idle times. In terms of improvements to the game, she expressed that it would be useful if the teacher could track the students' progress through the game, perhaps through an SMS message when they have completed the exercises.

\subsection{Student Feedback}

Once we had approval and feedback from the teachers, we collected feedback from the students in each user group.

\subsubsection{Primary School in Tanzania}

After incorporating teachers' comments and suggestions, the game was ready to be tested by the Mlimani students. Before testing with students, we added several questions to the game inspired by past exams taken by the students to make the questions familiar to them. This eliminated the risk of confusing the students with questions they might be unfamiliar with, and allowed us to assess the game's design, somewhat independently of its content. The study included 15 students in total: seven from Standard 3 (three girls and four boys), four from Standard 4 (two girls and two boys), and four from Standard 5 (two girls and two boys). We explicitly selected a mix of girls and boys from each grade in order to obtain a more representative sample of the student population. On average, this group scored $87 \%$ on their last English exam, with grades ranging from $67 \%$ to $100 \%$. We made sure to involve a diverse group of students with different skill levels so that we could understand how a variety of students reacted to the tool.

We asked teachers to first introduce the game and explain how it works to the students. Then, we assisted them in providing each student with a few minutes to interact with the technology and successfully answer one or two questions before passing the mobile phone to the next student. We observed students as they worked with the technology, as seen in Figure 8, and followed up with a few questions.

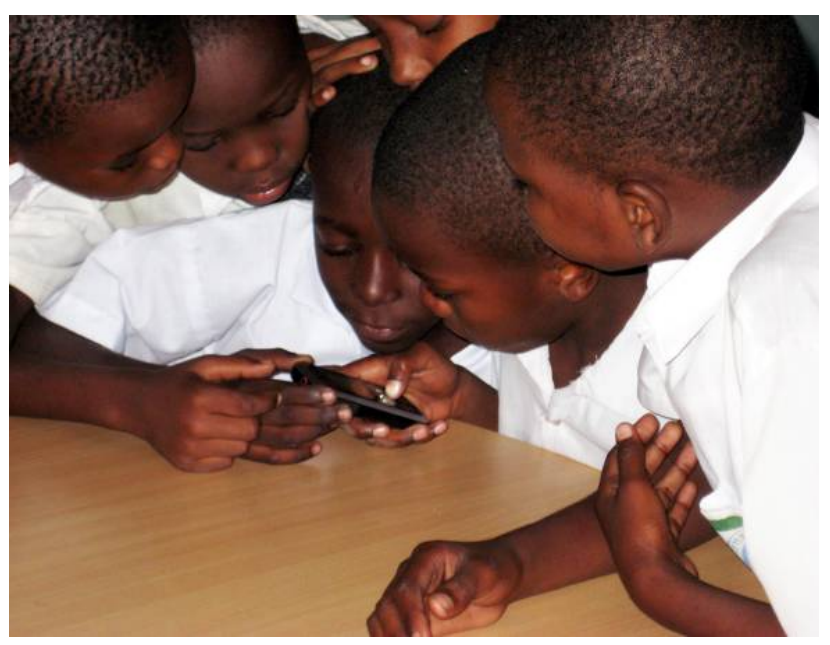

Figure 8. Students in Tanzania interacting with the mobile phone game.

During these user tests, we found that all of the students enjoyed using the mobile phone game and found it easy to use. As the teachers predicted, four to five students were able to share one phone screen, with one student selecting the answers using the phone's keypad and others observing. Sharing the phones in this way did not seem to trouble any of the students. Some students did not have prior experience using a phone so they took some time to get used to it; in particular, the continuously fading screen light (power saving) seemed to stump many of them. However, all students were able to quickly learn how to use the game. Receiving positive feedback for answering a question correctly was a tremendous motivation to the students to try hard. The "booing" audio feedback of the game for incorrect answers seemed to act as a disincentive to getting the answer wrong. We were concerned that it would also contribute to the weaker students becoming more insecure about playing the game, since other students laughed at students who repeatedly got booed. However, the competitive and football-loving attitude among students seemed to override any such insecurity. This audio feedback may therefore serve to encourage students to practice more so that they do not get booed as much in front of their peers. 
As expected, the older students arrived at the correct answers at a faster rate than younger students, but no student gave up on a question, even after multiple incorrect answers. The writing question proved to be challenging due to the difficulty in using the phone's keypad, but the teachers thought that having writing questions would be very useful to the students. Students were enthusiastic about getting another chance to play the game, and said they would play it at home as well, if they could. They also enjoyed the sound effects of the game and the multiple-choice format of the questions.

\subsubsection{Migrant Workers in Qatar}

The ROTA migrant workers were given the option to play the game twice a week in the last five or ten minutes of class, under the guidance of the instructors. Approximately $90 \%$ of the workers remained in class to participate in this pilot study. The workers were provided with phones they could use to play the game during the test sessions. This included 10 workers in the Beginner class and 12 workers in the Intermediate class. For the group of workers who were not enrolled in a structured English class consisting of 7 workers, testing took place three times a week for 30 minutes each under the guidance of one of our researchers. These workers were also provided with phones for testing and they all played the game for the entire duration of the test sessions.

All of the workers enjoyed the game and expressed interest in continuing to use the literacy tool. The workers not enrolled in a structured English class were especially excited to use a tool that would help them improve their English skills. Some workers found the questions difficult but nevertheless persevered in playing the game. Unsurprisingly, the adult learners put more emphasis on the educational value rather than the game component. The workers took the time to understand why they got a question wrong. Moreover, the workers who were not in a classroom setting frequently stopped to ask our researcher to explain why their answer to a question was incorrect. They would then make sure they understood the concept before answering the same question for the second time. This indicates that feedback for incorrect answers on the mobile phone game would be especially useful for students that are not in a structured English class.

As expected, after playing the game for some time, the workers found the audio feedback to be annoying and validated our decision to provide the option for toggling the audio feedback on and off. Some workers disliked the writing questions because they would spend time typing in long answers. Furthermore, they would get annoyed if they got a question wrong because they missed an apostrophe or some other element that they didn't consider to be important. There was also a lot of competition among the workers and they compared their scores among peers, in their attempt to get the highest score. This competitiveness was a strong motivator for the workers to perform better.

\subsubsection{School for the Deaf in the USA}

As described earlier, we conducted a pilot study with the students at WPSD using the content provided by the teacher. The teacher created questions based on known areas of difficulty faced by her English class students, such as noun-verb agreement, proper use of articles, verb tenses, etc. This provided the students with some familiar, but challenging content. The teacher also varied the level of difficulty for the questions chosen for each day's tests. The study included eighteen students in total: seven from the $7^{\text {th }}$ grade and eleven from the $8^{\text {th }}$ grade. Following the teacher's advice, we offered participation to all of the students from the $7^{\text {th }}$ and $8^{\text {th }}$ grade who were in that teachers' class, except for those students who had other significant learning challenges. We provided each participating student with a log sheet where they tracked the time they spent on the game, their score, and any observations or comments based on their experience with the phone game each day. Each student was given access to a phone so they could play the game simultaneously.

Researchers from our team observed some of the early class sessions and showed the teacher how to address any application errors that occurred on the phones. Students were able to take on roles such as changing the question file for each new day of testing, and charging the phones at the end of each day of testing. We observed students as they worked with the technology during some of the testing days, we collected the log sheets and reviewed their written observations, and finally we attended class presentations where they offered us additional feedback in person. After their presentations we had the opportunity to ask several follow-up questions.

We found that all of the students enjoyed using the mobile phone game and found it easy to play. As expected, the students were interested in having more choices instead of just playing a soccer themed game. They suggested several other sports and topics they would like to see in future versions of the game. Many of the students wanted the game to be more interactive - for example providing opportunities for competition with their peers, or having the opportunity to aim the ball, rather than automatically scoring a goal if they answer correctly. However, it is unclear if these interactions will detract from the educational goal of the game.

All of the students had some familiarity with cell phones although some did not have their own phones. Therefore it was very easy for them to use the phones and to navigate to the menus to load the desired content file into the game. Receiving positive feedback for answering a question correctly was a strong motivator for students, and one suggestion was to use a variety of different forms of positive feedback in future versions of the game. Overall, the sounds, lights and vibration were appealing to most students although a few students thought it could be distracting at times. Since some of the students were Deaf, others had some hearing, and some used hearing aids or other devices, we had a variety of responses to the audio feedback. Some students who could hear the sounds enjoyed them, while others thought they were too loud and once again validated our decision to provide toggling of sound and volume control via the menu options. The students played the game until they had answered all 10 questions assigned for each day of testing, with varying completion times. They were enthusiastic about using future iterations of the mobile phone game, especially if there were more game features that incorporated their suggestions. Furthermore, they would welcome the option to play it at home or when traveling as well. 


\subsection{Summary of User Feedback}

Overall, teachers/instructors found the CAT very easy to use and thought it would be a useful tool for them. Question types that support the curriculum would encourage more teachers to use it. In fact, this tool can be used for other subjects as well.

The mobile phone game was tested on the three user groups, which have participants from different age groups, backgrounds, and with different needs. The primary school students enjoyed the game and found it extremely motivating to learn from the audio feedback. Adult learners gave more emphasis to the educational aspect. Students in the deaf school in the US found it engaging and educational, and felt motivated to use the game in and outside classroom to enhance their learning.

\section{CONCLUSIONS AND FUTURE WORK}

In this paper, we have presented a mobile phone and web based solution to enhance the instruction of English literacy for both teachers and students in several underserved communities. Our literacy tool has two components: (1) an online content authoring tool for teachers, and (2) a mobile phone educational game that uses the content created by the teachers. The online content authoring enables collaborative content creation and repeated use of content for multiple classes. Further, the use of computing tools seemed to add motivation and encouragement for some teachers. Mobile phones are an ideal platform for the literacy game; especially in developing communities. Being portable and battery operated are some of the main advantage since access to a stable power grid is not always available. Even when computers are available in the classroom, using the literacy tool on the mobile phone can be less disruptive due to their portability. In order to maximize access to our English literacy tool, we plan to release it under an appropriate Open Source license so that interested users can benefit from the tool at no cost.

We are pursuing several avenues for enhancing our English literacy tool. The content authoring tool is being further enhanced to include several other features including a wider variety of question types, an enhanced interface, and greater flexibility in accommodating multiple users. Several games are also being created; some accessible on mobile phones, and others on traditional computers. The results presented here are from pilot projects conducted among user groups from different communities, backgrounds, age groups, and with different needs. Though the testing is still in an early stage, the idea of the CAT and the mobile phone game were well received by all groups and the results have improved with each study. Future work includes a long term study with quantitative measures of the effectiveness and impact of this literacy tool.

\section{ACKNOWLEDGMENTS}

The TechBridgeWorld research group is grateful to the many people and organizations that made this project possible. We would especially like to thank our main community partner during the iSTEP 2009 internship program, the University Computing Centre (UCC), who made it possible for us to conduct technology research projects in Tanzania, including our contacts Professor Beda Mutagahywa, Eric Beda Mutagahywa, Callista Duwe, Anthony Gesase, and Dorothy Mbonike. We would also like to acknowledge Vice Chancellor Professor Rwekaza Sympho
Mukandala, Saudin Jacob, and Dr. Tito Mwinuka from the University of Dar es Salaam for their assistance.

We are extremely grateful to the following partners who were involved with needs assessment, initial testing, and feedback for this project: Mlimani Primary School in Tanzania, teachers, students, and administrators; Western Pennsylvania School for the Deaf in the United States, including teacher Joyce Maravich, her students, and the school's administrators; and research participants and colleagues from Reach Out to Asia and Carnegie Mellon University in Qatar during this project.

Special thanks are due to several advisors to TechBridgeWorld's students who were involved in this research including: Yonina Cooper, David Dausey, Karumuna Kaijage, Theresa Kaijage, and Silvia Pessoa.

The research reported in this publication was supported in part by discretionary gifts to the TechBridgeWorld research group at Carnegie Mellon University, by internal funding sources at Carnegie Mellon University in Qatar, by the Qatar Foundation for Education, Science, and Community Development, by the support of National Priorities Research Program grant \#30-6-7-91 from the Qatar National Research Fund, by Carnegie Mellon University in Qatar, by the University Computing Centre, Ltd. located at the University of Dar es Salaam in Tanzania, and by the Holleran Scholars Fund. The opinions expressed are those of the authors and do not necessarily represent the views of any of our sponsors.

\section{REFERENCES}

[1] Abimbola, R., Alismail, H., Belousov, S., Dias, B., Dias, M. F., Dias, M. B., Fanaswala, I., Hall, B., Nuffer, D., Teves, E., Thurston, J. and Velázquez, A. 2009. iSTEP Tanzania 2009: Inaugural Experience. Technical Report. Carnegie Mellon University.

[2] Banerjee, A., Cole, S., Duflo, E. and Lindon, L. 2005. Remedying Education: Evidence from Two Randomized Experiments in India. NBER Working Paper No. 11904.

[3] Connected Limited Device Configurations (CLDC), JSR 139: http://java.sun.com/products/cldc.

[4] Horowitz, J., Sosenko, L., Hoffman, J., Ziobrowski, J., Tafoya, A., Haagenson, A., Hahn, S.: Evaluation of the PBS Ready to Learn Cell Phone Study: Learning Letters with Elmo. Final Report to the U. S. Department of Education (September 2006).

[5] Kent, R. D. 2004. Language of the Deaf: Acquisition of English. In the MIT Encyclopedia of Communication Disorders, 336-337.

[6] Light Weight User Interface Toolkit: https://lwuit.dev.java.net.

[7] McCoy, K. F. and Masterman, L. N. 1997. A Tutor for Teaching English as a Second Language for Deaf Users of American Sign Language. In Proceedings of Natural Language Processing for Communication Aids, an ACL/EACL97 Workshop (Madrid, Spain, July 12, 1997).

[8] Mills-Tettey, G. A. et al. 2009. Improving Child Literacy in Africa: Experiments with an Automated Reading Tutor. In Proceedings of the 3rd IEEE/ACM International Conference 
on Information and Communication Technologies and Development (Doha, Qatar, April 17 - 19, 2009). ICTD ‘09.

[9] Mobile Information Device Profile (MIDP), JSR 118: http://java.sun.com/products/midp.

[10] Mostow, J. 2008. Experience from a Reading Tutor that listens: Evaluation purposes, excuses, and methods. In C. K. Kinzer \& L. Verhoeven (Eds.), Interactive Literacy Education: Facilitating Literacy Environments Through Technology, pp. 117-148. New York: Lawrence Erlbaum Associates, Taylor \& Francis Group

[11] One Laptop per Child (OLPC): http://laptop.org/en/.
[12] Pawar, U. S., Pal, J. and Toyama, K. 2006. Multiple Mice for Computers in Education in Developing Countries. In Proceedings of the 1st IEEE/ACM International Conference on Information and Communication Technologies and Development (Berkeley, United States, May 25 - 26, 2006). ICTD '06.

[13] Revelle, G., Reardon, E., Makeda, M. G., Betancourt, J., and Jennifer, K. 2007. The Use of Mobile Phones to Support Children's Literacy Learning. In Lecture Notes in Computer Science (LNCS), 253-258. 